\title{
Increased Serum Dihomo- $\gamma$-linolenic Acid Levels Are Associated with Obesity, Body Fat Accumulation, and Insulin Resistance in Japanese Patients with Type 2 Diabetes
}

\author{
Yuya Tsurutani, Kosuke Inoue, Chiho Sugisawa, Jun Saito, \\ Masao Omura and Tetsuo Nishikawa
}

\begin{abstract}
:
Objective To clarify the associations between serum omega-6 (n-6) and omega-3 (n-3) polyunsaturated fatty acid (PUFA) levels and obesity-related metabolic abnormalities in patients with type 2 diabetes.

Methods and Materials Data from 225 Japanese patients with type 2 diabetes were cross-sectionally analyzed. The serum levels of n-6 PUFAs [dihomo- $\gamma$-linolenic acid (DGLA) and arachidonic acid (AA)] and n-3 PUFAs (eicosapentaenoic acid and docosahexaenoic acid) were measured, and the estimated $\Delta-5$ desaturase (D5D) activity was calculated based on the AA to DGLA ratio. The associations between the composition of PUFAs and obesity-related parameters, including the body mass index (BMI), waist circumference, alanine amino transferase (ALT) level, homeostatic model assessment of insulin resistance (HOMA-IR), and body fat percentage, as measured by a bioelectrical impedance analysis, were analyzed.

Results Among the PUFAs, the DGLA level had the strongest correlations with BMI $(\mathrm{p}<0.001)$, waist circumference $(\mathrm{p}<0.001)$, ALT level $(\mathrm{p}<0.001)$, HOMA-IR $(\mathrm{p}<0.001)$, and body fat percentage $(\mathrm{p}<0.01)$. AA was positively correlated and D5D was negatively correlated with several obesity-related parameters, while n3 PUFAs did not have a constant correlation. A multivariate regression analysis revealed that the DGLA level was an independent determinant for HOMA-IR $(\beta=0.195, \mathrm{p}=0.0066)$ after adjusting for sex, age, BMI, and the ALT, triglyceride, and HbAlc levels.

Conclusion A high serum DGLA level was associated with obesity, body fat accumulation, a high ALT level, and insulin resistance in patients with type 2 diabetes. The measurement of the serum PUFA levels may be useful for evaluating metabolic abnormalities and estimating the dietary habits of patients.
\end{abstract}

Key words: polyunsaturated fatty acid, dihomo- $\gamma$-linolenic acid, obesity, type 2 diabetes, insulin resistance

(Intern Med 57: 2929-2935, 2018)

(DOI: 10.2169/internalmedicine.0816-18)

\section{Introduction}

In the past half-century, the total and saturated fat intakes have fallen in industrial countriesr $(1,2)$. However, the ratio of omega-6 (n-6) to omega-3 (n-3) polyunsaturated fatty acids (PUFAs) has increased in parallel with the increase in the prevalence of obesity (3).

n-3 and n-6 PUFAs are essential fatty acids that must be supplied from the diet due to the lack of endogenous en- zymes for desaturation in humans. n-3 PUFAs are mainly supplied from fish oils, whereas n-6 PUFAs are broadly available from sources ranging from vegetable oils to meat products. $\mathrm{n}-3$ and $\mathrm{n}-6$ PUFAs compete for common metabolic enzymes (4). Basic studies have shown that $n-3$ and $n-$ 6 PUFAs have opposing effects on adipogenesis (5), the browning of adipose tissue (6), the brain-gut-adipose tissue axis (7), and systemic inflammation (8). Many epidemiological or clinical studies have shown that high $n-3 / n-6$ PUFA ratios were associated with a decreased incidence of 
ischemic heart disease $(9,10)$.

In addition, the association between PUFA profiles and obesity or its-related disorders have been attracting attention. A previous report on 534 participants of the Woman's Health Study showed that baseline red blood cell membrane phospholipid cis n-6 fatty acid levels were positively associated and cis n-3 FAs were inversely associated with longitudinal weight gain (11). Recent reports on the EPIC-InterAct Case-Cohort Study (12) and Hitachi Health Study (13) showed that among the n-3 and n-6 PUFAs, the dihomo- $\gamma$ linolenic acid (DGLA) level had the strongest positive association with the incidence of type 2 diabetes. In addition, a cross-sectional report showed that a high DGLA level and low $\Delta-5$ desaturase (D5D) activity, a rate-limiting enzyme involved in the formation of arachidonic acid (AA) from DGLA, were associated with hepatic steatosis (14). These previous findings suggest that PUFA metabolism-particularly in n-6 PUFA-is closely associated with obesity and itsrelated disorders. However, most previous studies have included participants without diabetes at baseline, and there have been few studies limited to patients with type 2 diabetes. In particular, the clinical data that have been used to analyze the association between PUFA profiles and multiple metabolic abnormalities in patients with type 2 diabetes are limited. In the present study, we aimed to clarify the associations between the composition of PUFAs and multiple parameters related to obesity-related metabolic abnormalities.

\section{Materials and Methods}

\section{Data sources and participants}

We performed a retrospective, cross-sectional study using clinical records from 225 patients (male, $n=165$; female, $n=$ 60) who were diagnosed with type 2 diabetes according to the Japan Diabetes Society guidelines (15). The participants were admitted to the Endocrinology and Diabetes Center at Yokohama Rosai Hospital for glycemic control and diabetes education from January 2011 to December 2016. The exclusion criteria were treatment with eicosapentaenoic acid (EPA), docosahexaenoic (DHA), ezetimibe or insulin; renal insufficiency, with an estimated glomerular filtration rate (eGFR) of $<30 \mathrm{~mL} / \mathrm{min} / 1.73 \mathrm{~m}^{2}$; decreased insulin secretion with a fasting serum C-peptide immunoreactivity (CPR) level of $<0.17 \mathrm{nmol} / \mathrm{L}(=0.5 \mathrm{ng} / \mathrm{dL})$; a history of diabetesrelated ketoacidosis and diabetes-related coma within 6 months; a history of severe infection or trauma requiring hospital care within 6 months; malignant neoplasm (treated or untreated); or a history of surgery for weight loss. This study was carried out in accordance with the ethical principles of the Declaration of Helsinki and was approved by the research ethics committee of Yokohama Rosai Hospital (Approved ID: 28-58). The need for informed consent was waived because of the retrospective nature of the study and because the study utilized data provided in the course of normal patient care.

\section{Measurements and the calculation of indices}

The history of diabetes was self-reported. At admission, the participant's weight and height were measured and were used to calculate body mass index (BMI). The waist circumference measurement was performed by the investigator at the level of the umbilicus, directly on the skin while the participant was standing erect. On the second morning of hospitalization, blood samples were collected after over 12 hours of fasting. The blood samples were collected in serum tubes containing separating gel and were centrifuged at 1785 $\times \mathrm{g}$ for $7 \mathrm{~min}$. The supernatants were then collected and immediately transported to SRL (Tokyo, Japan) at $4^{\circ} \mathrm{C}$. The serum PUFA (DGLA, AA, EPA, and DHA) levels were measured using gas chromatography-flame ionization detection. The estimated D5D activity was calculated as the ratio of AA to DGLA $(13,14)$. The glycated hemoglobin (HbA1c) level was measured by high-performance liquid chromatography (TOSOH, Tokyo, Japan). The immunoreactive insulin (IRI) level was measured by a chemiluminescent enzyme immunoassay. The homeostatic model assessment of insulin resistance (HOMA-IR) value was calculated as [fasting plasma glucose $(\mathrm{mg} / \mathrm{dL}) \times$ fasting IRI $(\mu \mathrm{U} / \mathrm{mL}) / 405]$. The body composition was measured in 77 patients by bioelectrical impedance analysis using an InBody 720 system (16) (Biospace, Tokyo, Japan), which is approved for use as a medical instrument by Pharmaceuticals and Medical Devices Agency, Japan. The body fat percentage and lean mass weight measured by the InBody 720 were strongly correlated with the values measured by dual-energy X-ray absorptiometry in obese patients $(r=0.736$ and $r=0.899)$ (17). The skeletal mass index was calculated as the appendicular skeletal muscle mass/height ${ }^{2}$ (18). The parameters of atherosclerosis were assessed based on brachial-ankle pulse wave velocity (baPWV) (19) and the maximum carotid intimamedia thickness (max IMT) (20).

\section{Statistical analysis}

The data are given as medians (interquartile range) because most of the continuous variables were nonparametrically distributed. Spearman's rank correlation coefficients were calculated to assess the relationships between continuous variables. The Mann-Whitney $U$ test was used for intergroup comparisons. Multiple regression analyses were performed using a least-squares analysis of variance to clarify the independent factors associated with BMI or HOMA-IR. The independent variables included in the multiple regression analysis for BMI were participant sex, age, current smoking, habitual drinking, triglyceride (TGL) level, HbA1c level, and the PUFA levels that were significantly correlated with the BMI in a simple correlation analysis. The independent variables included in the multiple regression analysis for HOMA-IR were participant sex, age, BMI, eGFR, ALT level, TGL level, HbA1c level, and the PUFA levels that were significantly correlated with the indices of 
insulin resistance in the simple correlation analysis. Missing values were excluded from the analysis. In all of the statistical analyses, $\mathrm{p}$ values of $<0.05$ were considered to indicate statistical significance. The JMP ${ }^{\circledR} 12$ software program (SAS Institute, Cary, USA) was used for all of the analyses.

Table 1. The Clinical Characteristics of the Patients.

\begin{tabular}{|c|c|}
\hline Sex (male/female) & $165 / 60$ \\
\hline Age (years) & $63(49-72)$ \\
\hline Duration of diabetes (years) & $6(0-13)$ \\
\hline BMI $\left(\mathrm{kg} / \mathrm{m}^{2}\right)$ & $25.7(22.8-28.8)$ \\
\hline Waist circumference $(\mathrm{cm})$ & $92.0(87.0-100.0)$ \\
\hline $\mathrm{HbA1c}(\%)$ & $9.8(8.4-11.2)$ \\
\hline $\operatorname{ALT}(\mathrm{IU} / \mathrm{L})$ & $25.0(18.0-39.0)$ \\
\hline Total-cholesterol (mg/dL) & $163(145-189.5)$ \\
\hline HDL-cholesterol (mg/dL) & $43(37-50.5)$ \\
\hline TGL (mg/dL) & $121(96-156)$ \\
\hline Non-HDL-cholesterol (mg/dL) & $121(100.5-145.5)$ \\
\hline eGFR (mL/min/1.73m²) & $77.8(67.3-89.3)$ \\
\hline HOMA-IR & $2.10(1.24-3.44)$ \\
\hline DGLA $(\mu \mathrm{g} / \mathrm{mL})$ & $36.3(28.2-47.4)$ \\
\hline $\mathrm{AA}(\mu \mathrm{g} / \mathrm{mL})$ & $190.5(157.0-235.3)$ \\
\hline EPA $(\mu \mathrm{g} / \mathrm{mL})$ & $55.0(34.5-75.7)$ \\
\hline DHA $(\mu \mathrm{g} / \mathrm{mL})$ & $133.7(106.5-171.5)$ \\
\hline EPA/AA & $0.29(0.17-0.43)$ \\
\hline Estimated D5D & $5.0(4.2-6.7)$ \\
\hline Body fat percentage $(\%)$ & $31.7(22.3-37.9)$ \\
\hline Skeletal mass index & $7.2(6.7-7.9)$ \\
\hline Current smoking, $\mathrm{n}(\%)$ & $69(30.7 \%)$ \\
\hline Habitual drinking, $\mathrm{n}(\%)$ & $122(47.8 \%)$ \\
\hline History of cerebrovascular disease, $\mathrm{n}(\%)$ & $30(13.3 \%)$ \\
\hline Number of glucose-lowering agents used & $1(1-3)$ \\
\hline Taking lipid-lowering agents, n (\%) & $89(39.5 \%)$ \\
\hline Taking antihypertensive agents, $\mathrm{n}(\%)$ & $108(46.7 \%)$ \\
\hline
\end{tabular}

Data are given as medians (interquartile range). BMI: body mass index, HbA1c: glycated hemoglobin, ALT: alanine amino transferase, HDL: high-density lipoprotein, TGL: triglycerides, eGFR: estimated glomerular filtration rate, HOMA-IR: homeostatic model assessment of insulin resistance, DGLA: dihomo- $\gamma$-linolenic acid, AA: arachidonic acid, EPA: eicosapentaenoic acid, DHA: docosahexaenoic acid, D5D: $\Delta-5$ desaturase

\section{Results}

\section{The clinical characteristics of the patients}

The characteristics of the 225 cases in this study are shown in Table 1 . The median age was 63 (49-72) years, and $165(73.3 \%)$ patients were male. Nearly half of the patients $(45.3 \%)$ were obese $\left(\mathrm{BMI} \geq 25 \mathrm{~kg} / \mathrm{m}^{2}\right)$. The glycemic control was poor, with a mean $\mathrm{HbA1c}$ level of $9.8 \%$. Eighty-nine patients $(39.5 \%)$ were taking lipid-lowering agents (statins or fibrates). The median lipid levels were almost within the normal range. The correlations of each of the serum PUFAs are shown in Table 2. No inverse correlation was observed between the levels of n-3 and n-6 PUFAs.

\section{Correlations between the PUFA composition and clinical parameters}

To clarify whether n-6 or n-3 PUFAs were associated with obesity-related parameters, we analyzed the correlations between the PUFA composition and various clinical parameters. As shown in Table 3, among the PUFAs, the DGLA level had the strongest correlations with BMI $(\rho=0.316, p<$ $0.001)$, waist circumference $(\rho=0.227, p<0.001)$, ALT level $(\rho=0.270, p<0.001)$, HOMA-IR $(\rho=0.367, p<0.001)$, and body fat percentage $(\rho=0.315, p<0.01)$. AA was significantly correlated with BMI $(\rho=0.217, p=0.0010)$, waist circumference $(\rho=0.165, p=0.020)$, ALT level $(\rho=0.150, p=0.025)$, and HOMA-IR $(\rho=0.169, p=0.022)$; however, the correlations were weaker than those of DGLA. The estimated D5D activity had negative correlations with BMI $(\rho=-0.166, p=$ 0.013), ALT level $(\rho=-0.178, p=0.0076)$, and HOMA-IR ( $\rho$ $=-0.255, p<0.001)$. EPA $(\rho=-0.159, p=0.017)$, EPA/AA ratio $(\rho=-0.246, p<0.001)$, and estimated D5D activity $(\rho=-0.166$, $\mathrm{p}=0.013$ ) were negatively associated with BMI. Age had a negative correlation with n-6 PUFAs and positive correlations with n-3 PUFAs and the EPA/AA ratio. Age also had a negative correlation with BMI $(\rho=-0.429, p<0.001)$. A multivariate regression analyses adjusted for sex, age, current smoking, habitual drinking, TGL level, and HbA1c level, revealed that the DGLA and AA levels were the only independent determinants of BMI (Table 4). After adjusting for sex, age, BMI, and eGFR, we observed no significant asso-

Table 2. The Correlations of Serum PUFAs.

\begin{tabular}{lcccccc}
\hline & DGLA & AA & EPA & DHA & EPA/AA & Estimated D5D \\
\hline DGLA & - & $0.446^{* * *}$ & -0.101 & 0.099 & $-0.300^{* * *}$ & $-0.681^{* * *}$ \\
AA & & - & $0.164^{*}$ & $0.305^{* * *}$ & $-0.262^{* * *}$ & $0.293^{* * *}$ \\
EPA & & & - & $0.810^{* * *}$ & $0.892^{* * *}$ & $0.250^{* * *}$ \\
DHA & & & & - & $0.645^{* * *}$ & $0.143^{*}$ \\
EPA/AA & & & & & - & 0.128 \\
Estimated D5D & & & & & & - \\
\hline
\end{tabular}

Spearman correlation coefficient $(\rho)$ were used to evaluate correlations between variables. $* \mathrm{p}<0.05$, $* * \mathrm{p}<0.01, * * * \mathrm{p}<0.001$. PUFAs: polyunsaturated fatty acids, DGLA: dihomo- $\gamma$-linolenic acid, AA: arachidonic acid, EPA: eicosapentaenoic acid, DHA: docosahexaenoic acid, D5D: $\Delta-5$ desaturase 
Table 3. Correlations between PUFA Levels and Clinical Parameters.

\begin{tabular}{lcccccc}
\hline & DGLA & AA & EPA & DHA & EPA/AA & Estimated D5D \\
\hline BMI & $0.316 * * *$ & $0.217 * *$ & $-0.159 *$ & -0.074 & $-0.246 * * *$ & $-0.166 *$ \\
Age & $-0.260 * * *$ & $-0.328 * * *$ & $0.324 * * *$ & $0.253 * * *$ & $0.454 * * *$ & 0.033 \\
Waist circumference & $0.227 * * *$ & $0.165 *$ & -0.088 & -0.003 & $-0.152 *$ & -0.109 \\
ALT level & $0.270 * * *$ & $0.150 *$ & -0.078 & 0.008 & $-0.147 *$ & $-0.178 * *$ \\
TGL & $0.362 * * *$ & $0.270 * * *$ & -0.044 & $0.234 * * *$ & $-0.169 *$ & $-0.151 *$ \\
HbA1c level & -0.114 & 0.047 & $-0.136 *$ & -0.135 & $-0.157 *$ & 0.125 \\
HOMA-IR & $0.367 * * *$ & $0.169 *$ & -0.012 & 0.064 & -0.097 & $-0.255^{* * *}$ \\
Body fat percentage & $0.315 * *$ & 0.208 & -0.101 & 0.013 & -0.188 & -0.182 \\
Skeletal mass index & 0.031 & 0.100 & $-0.229 *$ & $-0.272 *$ & $-0.274 *$ & 0.030 \\
\hline
\end{tabular}

Spearman correlation coefficient $(\rho)$ were used to evaluate correlations between variables. $* \mathrm{p}<0.05, * * \mathrm{p}<0.01$, $* * * \mathrm{p}<0.001$. FUFA: polyunsaturated fatty acid, BMI: body mass index, ALT: alanine amino transferase, TGL: triglyceride, HbA1c: glycated hemoglobin, HOMA-IR: homeostatic model assessment of insulin resistance, DGLA: dihomo- $\gamma-$ linolenic acid, AA: arachidonic acid, EPA: eicosapentaenoic acid, DHA: docosahexaenoic acid, D5D: $\Delta-5$ desaturase

Table 4. Multivariate Associations of Clinical Variables with Body Mass Index.

\begin{tabular}{|c|c|c|c|c|c|c|c|c|c|c|}
\hline \multirow{4}{*}{ Measure } & \multicolumn{10}{|c|}{ Body mass index } \\
\hline & \multicolumn{10}{|c|}{ Multivariate model } \\
\hline & \multicolumn{2}{|c|}{ Model 1} & \multicolumn{2}{|c|}{ Model 2} & \multicolumn{2}{|c|}{ Model 3} & \multicolumn{2}{|c|}{ Model 4} & \multicolumn{2}{|c|}{ Model 5} \\
\hline & $\beta$ & $\mathrm{p}$ & $\beta$ & $\mathrm{p}$ & $\beta$ & $\mathrm{p}$ & $\beta$ & $\mathrm{p}$ & $\beta$ & $\mathrm{p}$ \\
\hline Sex (female) & -0.006 & 0.92 & -0.004 & 0.95 & 0.020 & 0.75 & 0.009 & 0.88 & 0.013 & 0.83 \\
\hline Age & -0.407 & $<0.0001$ & -0.397 & $<0.0001$ & -0.433 & $<0.0001$ & -0.416 & $<0.0001$ & -0.443 & $<0.0001$ \\
\hline Current smoking (yes) & -0.078 & 0.20 & -0.060 & 0.32 & -0.069 & 0.26 & -0.071 & 0.25 & -0.074 & 0.23 \\
\hline Habitual drinking (yes) & -0.088 & 0.14 & -0.104 & 0.062 & -0.101 & 0.10 & -0.101 & 0.10 & -0.092 & 0.13 \\
\hline $\mathrm{HbA1c}$ & -0.131 & 0.028 & -0.153 & 0.0094 & -0.161 & 0.0074 & -0.164 & 0.0062 & -0.146 & 0.015 \\
\hline TGL & 0.114 & 0.076 & 0.123 & 0.050 & 0.167 & 0.0063 & 0.162 & 0.0080 & 0.158 & 0.010 \\
\hline DGLA & 0.153 & 0.022 & & & & & & & & \\
\hline AA & & & 0.148 & 0.025 & & & & & & \\
\hline EPA & & & & & -0.033 & 0.60 & & & & \\
\hline EPA/AA ratio & & & & & & & -0.066 & 0.31 & & \\
\hline Estimated D5D & & & & & & & & & -0.062 & 0.31 \\
\hline $\mathrm{R}^{2}$ & 0.275 & & 0.273 & & 0.258 & & 0.260 & & 0.260 & \\
\hline $\mathrm{p}$ & $<0.0001$ & & $<0.0001$ & & $<0.0001$ & & $<0.0001$ & & $<0.0001$ & \\
\hline
\end{tabular}

$\rho$ : Spearman correlation coefficient, $\beta$ : standard partial regression coefficient. HbA1c: glycated hemoglobin, TGL: triglyceride, DGLA: dihomo- $\gamma$-linolenic acid, AA: arachidonic acid, EPA: eicosapentaenoic acid, D5D: $\Delta-5$ desaturase

ciation between the PUFA composition and the parameters of atherosclerosis, including the baPWV and max IMT values (data now shown). In addition, there was no significant association between the use of glucose-lowering agents and the PUFA levels (data not shown).

\section{The multivariate analysis of the association between PUFA levels and HOMA-IR}

We performed a multivariate regression analysis to clarify whether the DGLA level and estimated the D5D activity were independent determinants of HOMA-IR. After adjusting for sex, age, BMI, eGFR level, ALT level, TGL level, and HbA1c level, the DGLA level and the estimated D5D activity were found to be independently associated with HOMA-IR ( $\beta=0.195, p=0.0066$ and $\beta=-0.147, p=0.024$ ). The AA level was not independently associated with HOMA-IR $(\beta=0.065, p=0.38)$ (Table 5). We confirmed similar results in a multivariate regression analysis using log-transformed HOMA-IR as a dependent variable (data not shown).

\section{Discussion}

The results of the present study showed that a high serum DGLA level were associated with obesity, body fat accumulation, a high ALT level, and insulin resistance in Japanese patients with type 2 diabetes. The measurement of the serum PUFA levels, especially the n-6 PUFA levels, may be useful in evaluating obesity-related metabolic abnormalities.

We found that the DGLA level had significant positive correlations with the body mass index, waist circumference, ALT level, HOMA-IR, and body fat percentage. In addition, the AA level was positively correlated with several obesityrelated parameters. These results suggest that an excessive intake of n-6 PUFAs is associated with obesity and its- 
Table 5. Univariate and Multivariate Associations of Clinical Variables with HOMA-IR.

\begin{tabular}{|c|c|c|c|c|c|c|c|c|}
\hline \multirow{4}{*}{ Measure } & \multicolumn{8}{|c|}{ HOMA-IR } \\
\hline & \multirow{2}{*}{\multicolumn{2}{|c|}{ Univariate model }} & \multicolumn{6}{|c|}{ Multivariate model } \\
\hline & & & \multicolumn{2}{|c|}{ Model 1} & \multicolumn{2}{|c|}{ Model 2} & \multicolumn{2}{|c|}{ Model 3} \\
\hline & $\beta$ & $\mathrm{p}$ & $\beta$ & $\mathrm{p}$ & $\beta$ & $\mathrm{p}$ & $\beta$ & $\mathrm{p}$ \\
\hline Sex (female) & & & 0.087 & 0.1935 & 0.100 & 0.14 & 0.107 & 0.11 \\
\hline Age & -0.092 & 0.270 & 0.163 & 0.0615 & 0.155 & 0.09 & 0.136 & 0.12 \\
\hline BMI & 0.349 & $<0.0001$ & 0.217 & 0.0033 & 0.223 & 0.0032 & 0.234 & 0.0016 \\
\hline eGFR & 0.079 & 0.290 & 0.034 & 0.67 & 0.045 & 0.58 & 0.049 & 0.535 \\
\hline ALT & 0.448 & $<0.0001$ & 0.334 & $<0.0001$ & 0.352 & $<0.0001$ & 0.342 & $<0.0001$ \\
\hline TGL & 0.235 & 0.0013 & 0.112 & 0.10 & 0.154 & 0.03 & 0.158 & 0.02 \\
\hline $\mathrm{HbA1c}$ & -0.099 & 0.18 & -0.001 & 0.98 & -0.030 & 0.66 & -0.001 & 0.99 \\
\hline DGLA & 0.349 & $<0.0001$ & 0.195 & 0.0066 & & & & \\
\hline AA & 0.221 & 0.0026 & & & 0.065 & 0.38 & & \\
\hline Estimated D5D & -0.216 & 0.0032 & & & & & -0.147 & 0.024 \\
\hline $\mathrm{R}^{2}$ & - & & 0.3219 & & 0.296 & & 0.313 & \\
\hline $\mathrm{p}$ & - & & $<0.0001$ & & $<0.0001$ & & $<0.0001$ & \\
\hline
\end{tabular}

$\rho$ : Spearman correlation coefficient, $\beta$ : standard partial regression coefficient. HOMA-IR: homeostatic model assessment of insulin resistance, BMI: body mass index, eGFR: estimated glomerular filtration rate, ALT: alanine amino transferase, TGL: triglyceride, HbA1c: glycated hemoglobin, DGLA: dihomo- $\gamma$-linolenic acid, AA: arachidonic acid, D5D: $\Delta-5$ desaturase

related metabolic abnormalities. The intake of $n-6$ PUFAs is mainly derived from linoleic acid (LA), which is plentiful in vegetable oils. LA is metabolized to $\gamma$-linoleic acid (GLA) by $\Delta-6$ desaturase, and then progressively elongated to DGLA. GLA and DGLA are hardly supplied from a normal diet. Thus, high serum DGLA is usually associated with the excessive intake of LA. DGLA itself is thought to have an anti-inflammatory effect $(21,22)$. However, DGLA is further converted to AA, which promotes body fat gain through the mechanisms of adipogenesis $(5,23)$, lipid homoeostasis (24-26), and the brain-gut-adipose tissue axis (7, 27-29). In addition, AA-derived prostaglandin E2 and leukotriene B 4 have pro-inflammatory properties (21). Thus, the excessive intake of LA contributes to the development of obesity and a chronic inflammatory state via AA (30). In addition, AA is supplied directly from the diet, including grain-fed animals, dairy, and eggs. Patients with type 2 diabetes should avoid the high intake of n-6 PUFAs, including LA and AA, for the control of body weight and the prevention of cardiovascular disease. By checking the serum levels of PUFAs, physicians can estimate the dietary habits of patients and provide appropriate dietary advice.

The present study showed that the DGLA level had a stronger association with obesity-related parameters than that of AA. It may be related to the downregulated activity of D 5D, an enzyme converting DGLA to AA. The estimated D5 D activity is downregulated in individuals with obesity or insulin resistance $(31,32)$. Actually, the DGLA levels and estimated D5D activity tended to show mirror images with regard to the obesity-related parameters in the present study. In addition, the estimated activity of D6D, an enzyme converting GLA to DGLA, was shown to be positively correlated with obesity or insulin resistance (31). Unfortunately, the GLA level was not measured in the present study, and we could not calculate the estimated D6D activity. Overall, the DGLA level tends to increase in the state of obesity or insulin resistance. DLGA level may be a representative marker reflecting not only an excessive intake of n-6 PUFAs, but also obesity-related metabolic abnormalities.

There was no consistent correlation between the serum levels of n-3 PUFAs and obesity-related parameters. EPA had a significant negative correlation with the BMI; however, this association was diminished after adjusting for potential confounders. Previous studies showed that-in contrast to n-6 PUFAs-n-3 PUFAs have protective effects against the development of obesity (33). However, clinical intervention studies to examine the relationship between n-3 supplementation and the body composition have reported conflicting results $(34,35)$. One possible reason for the ambiguous results of $n-3$ supplementation on obesity is that $n-3$ supplementation in most interventional studies was performed without restricting the n-6 PUFA intake. In our study, there was no inverse correlation between the serum levels of $n-3$ and n-6 PUFAs (Table 2), suggesting that patients with a high intake of n-3 PUFAs did not always have a reduced intake of n-6 PUFAs. Hence, n-3 PUFA supplementation may not be effective if it is performed without restricting the $n-6$ PUFA intake.

In the present study, we only measured the levels of four serum fatty acids. This method is associated with some limitations. First, there was no information about the levels of fatty acids other than DGLA, AA, EPA, and DHA. In addition, the total food and fat intakes were not assessed. Thus, we could not determine whether the serum DGLA levels were directly associated with obesity-related parameters or if the association was intermediated by the other serum fatty 
acids. We assumed that in comparison to other fatty acids, DGLA has a stronger association with obesity-related parameters, because high DGLA levels showed the strongest positive association with the incidence of type 2 diabetes among the various types of fatty acids that were assessed in the EPIC-InterAct Case-Cohort Study (12) and the Hitachi Health Study (13). Second, serum samples were used in this study. The fatty acid composition in serum can be affected by the dietary fatty acid intake patterns of the previous few days (36). By measuring the fatty acid composition of the erythrocyte membrane or blood phospholipids, which reflect the meals of the previous several weeks (37), the associations could be more accurately assessed. Considering the significant correlation between the fatty acid levels of serum and red blood cells in healthy Japanese subjects (38), we do not believe that there was a large margin of error. Third, we used the absolute values of the fatty acid levels in our analysis; these values might have been affected by the levels of triglycerides or the amount of body fat. An analysis using the molar percentage of each fatty acid may attenuate the influence of these factors. In this study, multivariate analyses showed that the DGLA levels were a significant determinant of both the BMI and HOMA-IR values, independent from the triglyceride levels. We did not select body fat percentage as an independent variable for the multivariate analysis, because of the limited number of patients whose body composition was measured. We cannot deny that the associations between the DGLA levels and the BMI and HOMA-IR values are influenced by the amount of body fat.

The strength of the present study is that it analyzed the associations between PUFA levels and metabolic abnormalities by checking multiple clinical parameters. Few studies have investigated the association between the PUFA levels and the body composition of patients with type 2 diabetes. However, the present study is associated with several limitations in addition to the abovementioned limitations. First, this study employed a cross-sectional design. Thus, it is difficult to show a causal relationship between the PUFA composition and obesity-related metabolic abnormalities. Prospective studies are needed to confirm the results of the present study. Second, the amount of exercise was not assessed in this study. In addition, the amount of smoking and drinking was not quantitatively assessed. A self-administered semi-quantitative questionnaire could be used to estimate these parameters. Third, the participants were limited to inpatients with uncontrolled glucose levels, and a median HbA1c level of $9.8 \%$. The data from patients with wellcontrolled glucose levels were limited. We selected inpatients as participants because we attempted to collect various clinical parameters. We think that hospitalization had little influence on the circulating serum PUFA levels because the blood samples were collected on the second morning of hospitalization.

In conclusion, the results of the present study showed that a high serum DGLA level was associated with obesity, body fat accumulation, a high ALT level, and insulin resistance, in
Japanese patients with type 2 diabetes. The measurement of the serum PUFA levels may be useful in evaluating for obesity-related metabolic abnormalities and estimating the dietary habits of the patients.

The authors state that they have no Conflict of Interest (COI).

\section{Financial Support}

This work was partly supported by a Grant for Research on Intractable Diseases from the Japanese Ministry of Health, Labour and Welfare.

\section{Acknowledgement}

We thank Kaori Shimizu and Yuko Tada for their assistance with data acquisition.

\section{References}

1. Troiano RP, Briefel RR, Carroll MD, Bialostosky K. Energy and fat intakes of children and adolescents in the united states: data from the national health and nutrition examination surveys. Am J Clin Nutr 72: 1343S-1353S, 2000.

2. Swan G. Findings from the latest National Diet and Nutrition Survey. Proc Nutr Soc 63: 505-512, 2004.

3. Ailhaud G, Massiera F, Weill P, Legrand P, Alessandri JM, Guesnet P. Temporal changes in dietary fats: role of $n-6$ polyunsaturated fatty acids in excessive adipose tissue development and relationship to obesity. Prog Lipid Res 45: 203-236, 2006.

4. Simopoulos AP. Essential fatty acids in health and chronic disease. Am J Clin Nutr 70: 560S-569S, 1999.

5. Amri EZ, Ailhaud G, Grimaldi P. Fatty acids as signal transducing molecules: involvement in the differentiation of preadipose to adipose cells. J Lipid Res 35: 930-937, 1994.

6. Pisani DF, Amri EZ, Ailhaud G. Disequilibrium of polyunsaturated fatty acids status and its dual effect in modulating adipose tissue development and functions. OCL 22: D405, 2015.

7. Schwinkendorf DR, Tsatsos NG, Gosnell BA, Mashek DG. Effects of central administration of distinct fatty acids on hypothalamic neuropeptide expression and energy metabolism. Int $\mathrm{J}$ Obes $\mathbf{3 5}$ : 336-344, 2011.

8. James MJ, Gibson RA, Cleland LG. Dietary polyunsaturated fatty acids and inflammatory mediator production. Am J Clin Nutr 71: 343S-348S, 2000.

9. Dyerberg J, Bang HO, Stoffersen E, Moncada S, Vane JR. Eicosapentaenoic acid and prevention of thrombosis and atherosclerosis? Lancet 2: 117-119, 1978.

10. Nakamura T, Azuma A, Kuribayashi T, Sugihara H, Okuda S, Nakagawa M. Serum fatty acid levels, dietary style and coronary heart disease in three neighbouring areas in Japan: the Kumihama study. Br J Nutr 89: 267-272, 2003.

11. Wang L, Manson JE, Rautiainen S, et al. A prospective study of erythrocyte polyunsaturated fatty acid, weight gain, and risk of becoming overweight or obese in middle-aged and older women. Eur J Nutr 55: 687-697, 2016.

12. Forouhi NG, Imamura F, Sharp SJ, et al. Association of plasma phospholipid n-3 and n-6 polyunsaturated fatty acids with type 2 diabetes: The EPIC-InterAct Case-Cohort Study. PLoS Med 13: 117, 2016

13. Akter S, Kurotani K, Sato M, et al. High serum phospholipid dihomo- $\gamma$-linoleic acid concentration and low $\Delta 5$-desaturase activity are associated with increased risk of type 2 diabetes among Japanese adults in the Hitachi Health Study. J Nutr 147: 15581566, 2017.

14. Matsuda M, Kawamoto T, Tamura R. Predictive value of serum 
dihomo- $\gamma$-linolenic acid level and estimated $\Delta-5$ desaturase activity in patients with hepatic steatosis. Obes Res Clin Pract 11: 34-43, 2017.

15. Seino Y, Nanjo K, Tajim N, et al. Report of the committee on the classification and diagnostic criteria of diabetes mellitus. J Diabetes Investig 1: 212-228, 2010.

16. Ogawa H, Fujitani K, Tsujinaka T, Imanishi K, Shirakata H, Kantani A, et al. InBody 720 as a new method of evaluating visceral obesity. Hepatogastroenterology 58: 42-44, 2011.

17. Faria SL, Faria OP, Cardeal MDA, Ito MK. Validation study of multi-frequency bioelectrical impedance with dual-energy X-ray absorptiometry among obese patients. Obes Surg 24: 1476-1480, 2014.

18. Han DS, Chang KV, Li CM, et al. Skeletal muscle mass adjusted by height correlated better with muscular functions than that adjusted by body weight in defining sarcopenia. Sci Rep 6: 19457, 2016.

19. Yamashina A, Tomiyama H, Takeda K, et al. Validity, reproducibility, and clinical significance of noninvasive brachial-ankle pulse wave velocity measurement. Hypertens Res 25: 359-364, 2002.

20. Tsivgoulis G, Vemmos K, Papamichael C, et al. Common carotid artery intima-media thickness and the risk of stroke recurrence. Stroke 37: 1913-1916, 2006.

21. Field AE, Willett WC, Lissner L, Colditz GA. Dietary fat and weight gain among women in the Nurses' Health Study. Obesity (Silver Spring) 15: 967-976, 2017.

22. Rubin D, Laposata M. Regulation of agonist-induced prostaglandin E1 versus prostaglandin E2 production. A mass analysis. J Biol Chem 266: 23618-23623, 1991.

23. Gaillard D, Négrel R, Lagarde M, Ailhaud G. Requirement and role of arachidonic acid in the differentiation of pre-adipose cells Biochem J 257: 389-397, 1989.

24. Hennig B, Watkins BA. Linoleic acid and linolenic acid: effect on permeability properties of cultured endothelial cell monolayers. Am J Clin Nutr 49: 301-305, 1989.

25. Jump DB, Clarke SD, Thelen A, Liimatta M. Coordinate regulation of glycolytic and lipogenic gene expression by polyunsaturated fatty acids. J Lipid Res 35: 1076-1084, 1994.

26. Clarke S, Turini M, Jump D. Polyunsaturated fatty acids regulate lipogenic and peroxisomal gene expression by independent mechanisms. Prostaglandins Leukot Essent Fatty Acids 57: 65-69, 1997.

27. Di Marzo V, Matias I. Endocannabinoid control of food intake and energy balance. Nat Neurosci 8: 585-589, 2005.

28. Cha MC, Jones PJ. Dietary fat type and energy restriction interactively influence plasma leptin concentration in rats. J Lipid Res 39: 1655-1660, 1998.

29. An WS, Son YK, Kim SE, et al. Association of adiponectin and leptin with serum lipids and erythrocyte omega-3 and omega-6 fatty acids in dialysis patients. Clin Nephrol 75: 195-203, 2011.

30. Naughton SS, Mathai ML, Hryciw DH, McAinch AJ. Linoleic acid and the pathogenesis of obesity. Prostaglandins Other Lipid Mediat 125: 90-99, 2016.

31. Warensjö E, Rosell M, Hellenius M-L, Vessby B, De Faire U, Risérus U. Associations between estimated fatty acid desaturase activities in serum lipids and adipose tissue in humans: links to obesity and insulin resistance. Lipids Health Dis 8: 37, 2009.

32. Vessby B, Gustafsson I-B, Tengblad S, Boberg M, Andersson A. Desaturation and elongation of fatty acids and insulin action. Ann NY Acad Sci 967: 183-195, 2002.

33. Wang L. Omega- 3 and omega- 6 fatty acids: role in body fat gain and development of obesity. North Am J Med Sci 8: 163-171, 2015.

34. Simopoulos AP. An increase in the Omega-6/Omega-3 fatty acid ratio increases the risk for obesity. Nutrients 8: 1-17, 2016.

35. Buckley JD, Howe PRC. Anti-obesity effects of long-chain omega3 polyunsaturated fatty acids. Obes Rev 10: 648-659, 2009.

36. Ma J, Folsom AR, Shahar E, Eckfeldt JH. Plasma fatty acid composition as an indicator of habitual dietary fat intake in middleaged adults. The Atherosclerosis Risk in Communities (ARIC) Study Investigators. Am J Clin Nutr 62: 564-567, 1995.

37. Subbaiah PV, Kaufman D, Bagdade JD. Incorporation of dietary $\mathrm{n}-3$ fatty acids into molecular species of phosphatidyl choline and cholesteryl ester in normal human plasma. Am J Clin Nutr 58: 360-368, 1993.

38. Yanagisawa N, Shimada K, Miyazaki T, et al. Polyunsaturated fatty acid levels of serum and red blood cells in apparently healthy Japanese subjects living in an urban area. J Atheroscler Thromb 17: 285-294, 2010.

The Internal Medicine is an Open Access journal distributed under the Creative Commons Attribution-NonCommercial-NoDerivatives 4.0 International License. To view the details of this license, please visit (https://creativecommons.org/licenses/ by-nc-nd/4.0/).

(C) 2018 The Japanese Society of Internal Medicine Intern Med 57: 2929-2935, 2018 